\title{
ORIGIN OF PROTOGALACTIC EDDIES
}

\author{
A. D. Chernin \\ A. F. Ioffe Physico-Technical Institute \\ Leningrad, USSR
}

A highly excited state of the cosmic medium might develop in the isotropic Big Bang starting with weak irrotational perturbations that were likely to be of entropy type. Supersonic matter currents are an indispensable feature of this state. Initial irrotational gas motions become vortical when they become supersonic. One can expect that this may provide a clue in galactic cosmogony (1970, Nature, 226, 440).

The most effective mechanisms of vorticity generation are related to violent interactions of currents of strongly supersonic matter involving intersections of shocks and so-called non-evolutionary processes. It is of particular interest that both of them are generally accompanied by tangential discontinuities.

When two gaseous masses, "clouds", come into contact with a supersonic velocity, a component of their initial relative velocity along the line of the centres of the masses leads to two shocks propagating in opposite directions from the surface of contact of the masses. A transverse component of the initial velocity makes the material of the clouds move with different tangential velocities on the two sides of the surface. Such a tangential discontinuity is absolutely unstable, and this "vortex sheet" transforms eventually into a turbulent layer by the two shock fronts.

It seems reasonable to suppose that gaseous proto-clusters which originated as turbulent layers of large mass evolved into clusters like Virgo with many rapidly rotating galaxies. The encouraging resemblance between rough estimates of the characteristics of the layers and the major parameters of rotating galaxies and clusters of galaxies appears naturally as a definite consequence of gas-dynamical phenomena in the proto-galactic medium. 


\section{DISCUSSION}

Peebles: D. Hawley and I found that the observed orientation of the long axes of galaxies are remarkably close to random. Is this difficult to reconcile with the idea that the momenta of galaxies were produced in shocks continuous over large scales?

Chernin: I do not believe the statistics are yet large enough and close pairs are excluded from these samples.

Jones: Are you sure that the tidal torques theory predicts antiparallel spins? I can certainly imagine contrary situations.

Chermin: I agree these situations can occur but only rarely. 